- ORIGINAL ARTICLE

Volume 9 Issue 42017

DOI: 10.21315/eimj2017.9.4.3

ARTICLE INFO

Submitted: $11-8-2017$

Accepted: 16-11-2017

Online: 29-12-2017

\title{
Development, Translation and Validation of Questionnaires for Diarrhoea and Respiratory- related IIInesses during Probiotic Administration in Children
}

\author{
Amy Sie-Yik Lau', Muhamad Saiful Bahri Yusoff ${ }^{2}$, Yeong-Yeh Lee², \\ Sy-Bing Choi' ${ }^{1}$ Faridah Rashid ${ }^{2}$, Normala Wahid ${ }^{3}$, Jin-Zhong Xiao ${ }^{4}$, \\ Min-Tze Liong ${ }^{1}$ \\ ${ }^{1}$ School of Industrial Technology, Universiti Sains Malaysia, Pulau \\ Pinang, MALAYSIA \\ ${ }^{2}$ School of Medical Sciences, Universiti Sains Malaysia, Kelantan, \\ MALAYSIA \\ ${ }^{3}$ Community Health Center, Universiti Sains Malaysia, Pulau Pinang, \\ MALAYSIA \\ ${ }^{4}$ Next Generation Science Institute, Morinaga Milk Industry Co. Ltd., \\ Zama, $\mathcal{F A P A N}$
}

To cite this article: Lau S-YA, Yusoff MSB, Lee Y-Y, Choi S-B, Rashid F, Wahid N, Xiao J-Z, Liong M-T. Development, translation and validation of questionnaires for diarrhoea and respiratoryrelated illnesses during probiotic administration in children. Education in Medicine Journal. 2017;9(4):19-30. https://doi.org/10.21315/eimj2017.9.4.3

To link to this article: https://doi.org/10.21315/eimj2017.9.4.3

\section{ABSTRACT}

Background: Gastrointestinal illnesses and respiratory-related illnesses are common among young children in Malaysia, especially those who are attending day care. During administration of probiotic, the occurences of gastrointestinal and respiratory-related illnesses can be reduced. These were observed by evaluation through a single questionnaire. However, currently no single tool exists to simultaneously evaluate the domains of gastrointestinal and respiratory-related illnesses among these young children. The current study aimed to develop a source questionnaire in English, translate and validate into the Malay. Methods: Relevant domains of gastrointestinal and respiratory-related illnesses were identified to generate items and formed a screening tool through literature reviews, focus groups and opinions of experts. Results: The developed Basic Demographic and Lifestyle Questionnaire (BDLQ) and Monthly Healthy Questionnaires (MHQ) showed item-level content validity index (I-CVI) of 0.99 and 0.97 , respectively, while the translated Malay versions showed I-CVI of 1.00 and 0.99 , respectively. Item-level face validity index (I-FVI) of 1.00 for both questionnaires were obtained from 30 respondents showing that the items were clear and comprehensible. Conclusion: This study showed good level of I-CVI and I-FVI in both developed questionnaires and their Malay translated versions. These tools in English and Malay were valid and thus reliable to be used for assessing gastrointestinal and respiratory-related illnesses in young children.

Keywords: Questionnaire development, Validation, Probiotic, Diarrhoea, Respiratory-related illnesses 


\section{INTRODUCTION}

Gastrointestinal illnesses among infants and young children are a worldwide concern, with over two million hospitalisations in children under five years of age for diarrhoea. Diarrhoea is commonly defined as three or more loose or watery stools in the last 24 hours. Respiratory illnesses are currently the most common acute diseases in children with no available effective treatments or prophylaxes. Symptoms related to respiratory-related illnesses include rhinitis, nasal blockage and sore throat which could be triggered by both allergic and/or non-allergic reactions (1, 2). In Malaysia, diarrhoea and seasonal respiratory illness are among the most common diseases found to affect children, with diarrhoea being identified as one of the top five leading causes of death in children aged 1-19 years in the 1970s-1980s (35). Children who are attending day care centres are at a 1.5-3.0 times higher risks of gastrointestinal and respiratory tract infections than those cared at home or in small family care groups (6). These facts highlight the need to identify the symptoms and other factors which correlate to the occurrence of the gastrointestinal and respiratory-related illnesses.

Probiotics are defined as "live microorganisms which when administered in adequate amounts confer a health benefit on the host" (7). They improve the intestinal microbial balance, boosting the body system to combat against diseases by affecting the immune system (6). Probiotics have been studied extensively for their effects in preventing and treating a multitude of conditions, including the treatment of lactose intolerance, allergies, respiratory-related illnesses, traveller's diarrhoea and the prevention and treatment of nosocomial diarrhoea (8). In several studies on probiotic prevention of acute diarrhoea in daycare centres worldwide, probiotics tested such as Lactobacillus $G G$, Bifidobacterium lactis, and Lactobacillus reuteri showed a clear evidence of efficacy to reduce diarrhoea-related symptoms in a straindependent and dose-dependent manner (9). In addition, children fed with probiotic L. rhamnosus $G G$ had fewer respiratory tract infections with complications (otitis media, sinusitis, bronchitis and pneumonia) and less prescribed antibiotic treatments (10). Based on literature reviews, all the relevant domains were combined for the development of a single questionnaire to evaluate the occurrence and prevalence of the gastrointestinal and respiratory-related illnesses among young children.

Five guidelines are routinely adopted in translation processes, which include preparation, translation, pretesting, revising and documentation (11). The first step in translation is to prepare a desired questionnaire or to develop some data collection instruments for translation. The actual translation process is then performed, followed by pretests of the developed questionnaires prior to actual data collections. Pretesting is carried out to identify concepts or constructs which are specific to a certain language or culture (emic). Subsequently, based on the pretest results, the questionnaire designers and translators are able to revise and make appropriate adjustments and amendments to avoid conceptual bias and to achieve construct equivalence which represents the same underlying latent concept in different languages. In the final stage of the translation process, final decisions on revisions are made and the translated documents are then finalised. All finalised decisions and revisions are documented.

There are five general sources of evidence to support the validity of any research tools; (i) content, where items of research tools completely represent the measured outcomes; (ii) response process, where subjects understand the items of research tools correctly; (iii) internal structure, where acceptable reliability and factor structure is demonstrated; (iv) relations to other variables, where correlation exists among other tools that measure similar outcomes; 
and (v) consequences, where interpretation of scores justify a difference (12). In this study, we investigated two sources of evidence for validity of the newly developed instrument, namely content (content validity) and response process (face validity).

\section{METHODS}

Two main phases were involved; (i) Phase I: Development of tools/instruments; and (ii) Phase II: Translation and validation (Figure 1). In the development stage, relevant contents and information related to diarrhoea and respiratory-related illnesses were identified, generated, wrote and assembled into a usable instrument. This was performed via several approaches, namely focus groups, expert opinions, clinical observation, theory, literature reviews and interviews (13). The subsequent translation and validation processes involved expert reviews on the developed contents, to achieve relevance and representativeness, and to omit vagueness (14). The finalised version was subsequently pretested among the targeted population through face validation, to justify clarity and comprehensibility of the constructed items. The study was approved by the Human Ethics Committee of Universiti Sains Malaysia (USM) (USM/JEPeM/14100354). This clinical trial was registered under ClinicalTrials.gov (NCT02434042).

\section{Phase I: Development and Validation of Questionnaires}

\section{Development of questionnaires}

To develop a new and comprehensive instrument, several approaches were applied. One of the approaches was through focus groups. It was described as a discussion carried out in a small group of informants comprised of six to twelve people who were guided by a facilitator to express their ideas and opinions. Those relevant ideas and opinions were chosen and further discussed to clear any ambiguity, irrelevant and unclear information (13). Information was also gathered from clinical observation to describe all the symptoms occurred during diarrhoea and respiratory-related illnesses.

Literature reviews were also used as an alternative source of information during development of the questionnaire. This was crucial for the construction of symptoms such as those occurring during respiratoryrelated illnesses namely rhinitis, nasal blockage, sore throat, cough, common cold and fever (15), and symptoms for acute diarrhoea namely fever, bloody stools and vomiting (16).

Upon gathering all related information, expert opinions were consulted. A group of experts including sponsor of the study, colleagues and clinicians reviewed the information. Consultation involved commenting on the questionnaire, determination of new and important information that were absent but needed, and/or removal of non-relevant information from the instrument, based on their extensive expertise in the field of medical and instrument development (14). Responses and feedback were collected, summarised and the questionnaire was revised. With this, a draft questionnaire in the source language (English) was produced.

\section{Content validity (English version)}

Content validity refers to the appropriateness of an instrument that enables it to adequately measure the targeted outcomes (17). With the developed questionnaire in source language (English), the instrument was first pretested among a small group of experts (10 experts), that are well versed in the English and have expertise on the contents of the questionnaire. Content validity aimed to identify the appropriateness of the content in the questionnaire, whereas language validity aimed to ensure the comprehensibility of wording, sentence, style and communication effects of the questionnaire $(11,18)$. All experts were required to independently 


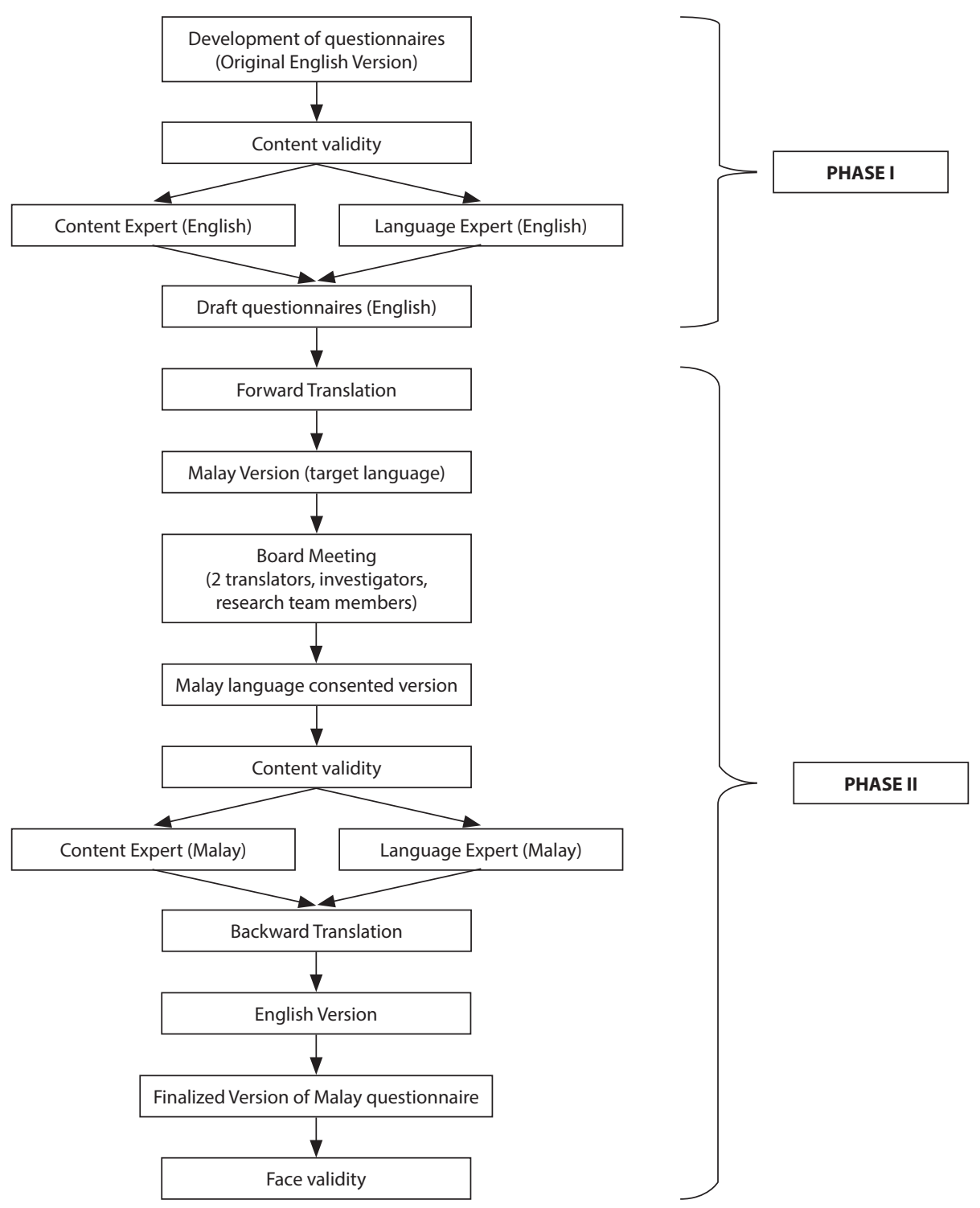

Figure 1: Schematic diagram of the development of questionnaire (Phase I) and translation and validation of questionnaire (Phase II).

rate each question and item with "YES" and "NO", where "YES" was given a score of one and "NO" was given a score of zero according to a binary scoring system (17, 19). Content validity index (CVI) was calculated based on the comments and feedbacks from the experts. CVI value was computed in two forms; I-CVI (Item-level Content Validity Index) for individual items, where the number of experts answering "YES" was divided by the number of total experts involved, and S-CVI (Scale-level Content Validity Index) for the overall scale, where I-CVI for all items are summed (19). S-CVI was computed in two ways, namely S-CVI/Ave (averaging calculation method) and S-CVI/UA (universal agreement calculation method). S-CVI/Ave was calculated from total I-CVI value divided by total number of items whereas S-CVI/ UA was calculated by getting the number of items which had $100 \%$ agreement and divided by the total number of items (19). An index value of 0.80 has been denoted as acceptable, with items of the content being valid and relevant (20). 


\section{Phase II: Translation and Validation of Questionnaires}

The translational process to produce questionnaires in two languages (source and target) utilised a decentering technique involving paraphrasing and translation between source and target languages $(21,22)$. In this study, forward- and backward-translations were applied in the translation process. The Basic Lifestyle and Demographic Questionnaire (BLDQ) and Monthly Health Questionnaire (MHQ) in English were translated into the Malay by two independent translators: a bilingual researcher and a qualified linguistic expert. Translation aimed at transferring the true meaning of the context from English to Malay while preserving the original meaning, style and effect of the text. In order to achieve high quality translation, the reliability, fluency and appropriateness of the translated instrument were heavily emphasised $(11,23)$.

\section{Forward translation}

Forward translation involved translating the English questionnaires into Malay (target language) by two certified independent bilingual translators. One of the translators was knowledgeable about health care terminologies and content of the questionnaires in both languages, while the other translator has such capabilities in addition to proficiency in informal phrases, slang and jargon, idiomatic expressions and emotional terms commonly used in the Malay community (23). These are needed to comprehensively envelop coherent medical aspects with cultural nuances of informal conversations. The translated Malay questionnaires were presented at board meeting, for comparison with the original English version to identify any ambiguities and discrepancies of words, sentences and meanings. Ambiguities and discrepancies were discussed and resolved using a committee-consensus approach to produce a reconciled version of the translated questionnaire. All revisions made were carefully documented in a reconciliation table showing the source language version, target language version, comments and modifications made.

\section{Content validity (Malay version)}

The translated questionnaire (in Malay) was subsequently pretested by another 10 experts who are well versed in the Malay and knowledgeable on the content of the questionnaire. The validation process was carried out as mentioned in Section Content Validity (English Version).

\section{Back translation}

Upon amendment, the translated questionnaires (Malay; target language) were subsequently translated backward into the source language (English). Two independent translators with qualifications as described in Section Forward Translation were recruited, with the exception that they are well-versed in English (source language) instead, to translate the Malay versions of questionnaires into English. They were completely blinded to the original version of the questionnaires and the questionnaires being back-translated (24). Two different versions of English-translated questionnaires were discussed and reconciled for any discrepancies and ambiguities. The Malay version was revised and modified. All revisions and modifications were done with consensus among all the translators, investigators and research team members. All revisions made were carefully documented in a reconciliation table showing the source language version, target language version, comments and modifications made. Final versions of questionnaires in English and Malay were finalised and subsequently pretested for face validity.

\section{Face validation}

Face validity is also known as surface validity, logical validity or appearance validity (25). It was carried out by the 
target group and technically untrained observers who determines the validity of the measurement procedure or domains.

Face validation assessment was carried out by 30 volunteers recruited from target population who will be responding to this instrument. A written informed consent was obtained from all the volunteers before assessment. Those recruited were parents and primary caregivers of preschool children. They evaluated the instructions, response format and the items of questionnaires for clarity. Similar to content validation, all 30 volunteers were required to independently rate each question and each item was rated according to a binary scoring system $(17,19)$. All feedbacks were documented in a reconciliation table, discussed and revised where needed, via consensus among the translators, investigators and research team members. Face validity index (FVI) was calculated to determine the quality of the translated questionnaires.

\section{RESULTS}

\section{Phase I: Development and Validation of Questionnaires}

\section{Development of questionnaire}

In developing the questionnaire, several methods were applied to gather information and ideas prior to constructing the domains for measurements of diarrhoea and respiratory-related illnesses among young children. Literature search indicated that symptoms commonly associated with respiratory-related illnesses were rhinitis, nasal blockage, sore throat, cough, common cold and fever. Discussions from focus group yielded additional symptoms such as runny nose, sneezing, wheezing, hoarseness, mucus production and vomiting.

Literature search on acute diarrhoea indicated that main symptoms include fever, bloody stools and vomiting. Similarly, discussions from focus group yielded additional symptoms such as abdominal pain, dehydration, nausea, loss of appetite, dizziness and rectal pain. Colour of stool during diarrhoea was also included in the questionnaire as colour changes may occur indicating other underlying causes of diarrhoea.

Initially, the administration of antibiotics was set as an exclusion criterion to exclude subjects who have administered antibiotics prior to the trial. However, based on opinions of experts, such an exclusion criterion was deemed unethical as children should not be forbidden to take antibiotics when it was prescribed and necessary. Thus, this exclusion criterion was removed. In addition, in the initial draft questionnaire, the duration (number of days) of symptoms for both diarrhoea and respiratory-related illnesses was not included. Upon discussion in focus group and opinions from experts, this information was deemed important and have thus been included.

\section{Content validity (English version)}

Contents of both questionnaires (BLDQ refer to S1 File; MHQ refer to S2 File) were validated for content and language by 10 experts. The BLDQ consisted of 38 items in four different sections (Table 1). Some minor modifications have been performed upon reconciliation of the draft questionnaires. In Section C (Child's health details), the phrase "Participant's health details" was changed into a more specific term "Child's health details". In Section D (Child's lifestyle), the phrase "Currently have pets or small animals at home" was changed to "Currently have pets or small animals at home? If yes, please state" for better clarify and specificity.

Content validation produced an overall validity index of over 0.80 , with a universal agreement index (S-CVI/UA) of 1.00 for both Sections A and B, and 0.91 and 0.86 for Sections $\mathrm{C}$ and $\mathrm{D}$, respectively. An average index (S-CVI/Ave) of 1.00 for Sections A and B, and 0.99 for Sections C and $\mathrm{D}$ were obtained (Table 1 ). 
The MHQ comprised of 72 items categorised into four sections (Table 2). An average index (S-CVI/Ave) of over 0.80 was obtained from all four sections. A universal agreement index (S-CVI/UA) of 0.40 was obtained for Section A, and 1.00, 0.98 and 0.83 for Sections B, B (I) and B (II), respectively.

\section{Phase II: Translation and Validation of Questionnaires}

\section{Forward and backward translation}

The reconciliation process indicated that the forward translated basic lifestyles and demographic questionnaire did not require further modifications during backtranslation (back to English). Five items in the monthly health questionnaire required minor modifications and rephrase during the reconciliation of forward translation. Meanwhile, no adjustment was needed in both questionnaires upon comparison between the back translated and the original English versions.

\section{Content validity (Malay version)}

Content validation was also conducted for the forward translated questionnaires (Malay version). In the BLDQ (S3 File), content validation produced an overall validity index of over 0.80, with a universal agreement index (S-CVI/UA) of 1.00 for all four Sections A, B, C and D. An average index (S-CVI/Ave) of 1.00 was obtained for all four sections as well (Table 3).

Meanwhile, an average index (S-CVI/Ave) of over 0.80 was obtained from all four sections for the MHQ (S4 File). A universal agreement index (S-CVI/UA) of 1.00 was obtained for section $\mathrm{A}$, and $0.50,0.93$ and 1.00 for sections B, B (I) and B (II), respectively (Table 4 ).

Table 1: Content validity index for Basic Lifestyle and Demographic questionnaire (English) by ten respondents

\begin{tabular}{lcccc} 
& \multirow{2}{*}{ Items } & \multicolumn{4}{c}{ Section } \\
\cline { 2 - 5 } & A & B & C & D \\
\hline S-CVI/Ave & 1.00 & 1.00 & 0.99 & 0.99 \\
S-CVI/UA & 1.00 & 1.00 & 0.91 & 0.86 \\
Average proportion of items & 1.00 & 1.00 & 0.99 & 0.99 \\
\hline
\end{tabular}

Note: S-CVI (Scale-level content validity index); UA (Universal agreement calculation method); Ave (Averaging calculation method); S-CVI/UA (Scale-level content validity index, universal agreement calculation method); S-CVI/Ave (Scale-level content validity index, averaging calculation method); Average proportion of items judged relevant across the ten respondents.

Table 2: Content validity index for Monthly Health Questionnaire (English) by ten respondents

\begin{tabular}{lcccc} 
& \multirow{2}{*}{ Items } & \multicolumn{4}{c}{ Section } \\
\cline { 2 - 5 } & A & B & B (I) & B (II) \\
\hline S-CVI/Ave & 0.88 & 1.00 & 0.99 & 0.93 \\
S-CVI/UA & 0.40 & 1.00 & 0.98 & 0.83 \\
Average proportion of items & 0.88 & 1.00 & 0.99 & 0.93 \\
\hline
\end{tabular}

Note: S-CVI (Scale-level content validity index); UA (Universal agreement calculation method); Ave (Averaging calculation method); S-CVI/UA (Scale-level content validity index, universal agreement calculation method); S-CVI/Ave (Scale-level content validity index, averaging calculation method); Average proportion of items judged relevant across the ten respondents. 
Table 3: Ratings on Item Scale by ten respondents for the Basic Lifestyle and Demographic Information questionnaires in English and translated questionnaires in Malay

\begin{tabular}{|c|c|c|c|c|c|c|c|c|c|c|}
\hline \multirow{3}{*}{ Items } & \multicolumn{10}{|c|}{ Sections } \\
\hline & \multicolumn{5}{|c|}{ Original English version } & \multicolumn{5}{|c|}{ Translated Malay version } \\
\hline & A & B & C & D & Overall & A & B & C & D & Overall \\
\hline S-CVl/Ave & 1.00 & 1.00 & 0.99 & 0.99 & 0.99 & 1.00 & 1.00 & 1.00 & 1.00 & 1.00 \\
\hline S-CVI/UA & 1.00 & 1.00 & 0.91 & 0.86 & 0.95 & 1.00 & 1.00 & 1.00 & 1.00 & 1.00 \\
\hline Average proportion of items & 1.00 & 1.00 & 0.99 & 0.99 & 0.99 & 1.00 & 1.00 & 1.00 & 1.00 & 1.00 \\
\hline
\end{tabular}

Note: S-CVI (Scale-level content validity index); S-CVI/UA (Scale-level content validity index, universal agreement calculation method); S-CVI/Ave (Scale-level content validity index, averaging calculation method); UA (Universal agreement calculation method); Ave (Averaging calculation method).

Table 4: Ratings on Item Scale by ten respondents for the Monthly Health Condition questionnaires in English and translated questionnaires in Malay

\begin{tabular}{|c|c|c|c|c|c|c|c|c|c|c|}
\hline \multirow{3}{*}{ Items } & \multicolumn{10}{|c|}{ Sections } \\
\hline & \multicolumn{5}{|c|}{ Original English version } & \multicolumn{5}{|c|}{ Translated Malay version } \\
\hline & A & B & B (I) & B (II) & Overall & A & B & B (I) & B (II) & Overall \\
\hline S-CVI/Ave & 0.88 & 1.00 & 0.99 & 0.93 & 0.97 & 1.00 & 0.90 & 0.99 & 1.00 & 0.99 \\
\hline S-CVI/UA & 0.40 & 1.00 & 0.98 & 0.83 & 0.90 & 1.00 & 0.50 & 0.93 & 1.00 & 0.93 \\
\hline Average proportion of items & 0.88 & 1.00 & 0.99 & 0.93 & 0.97 & 1.00 & 0.90 & 0.99 & 1.00 & 0.99 \\
\hline
\end{tabular}

Note: S-CVI (Scale-level content validity index); UA (Universal agreement calculation method); Ave (Averaging calculation method); S-CVI/UA (Scale-level content validity index, universal agreement calculation method); S-CVI/Ave (Scale-level content validity index, averaging calculation method).

\section{Face validity}

Face validation was performed using the finalised version of translated questionnaires with a total of 30 volunteers from a target population who were parents with preschool children and literate in the Malay. Similar to content validity, computation of face validity also included item-level face validity index (I-FVI), scale-level face validity index (S-FVI), universal agreement index (S-FVI/UA), averaging index (S-FVI/ Ave), and average proportion of items judged clear/comprehensible across 30 respondents. All items showed an index 1.00 for all the sections of all questionnaires studied (Table 5 and Table 6 ).

\section{DISCUSSION}

Cross-cultural translation and validation involves more than a literal translation which is crucial to produce a valid and suitable questionnaire or instrument, applicable in different target populations from different cultures and languages $(26,27)$. A new questionnaire or instrument can be adapted from the established ones or developed from scratch. Here, we developed a new questionnaire which was suitable to measure occurrences of diarrhoea and respiratoryrelated illnesses among children in Malaysia. During development of the questionnaires, ideas and contents were gathered from expert opinions, literature reviews and discussions in a focus group.

The new questionnaires drafted in the source language (English) were translated forward and backward. Translation process was challenging as the words in the source language have no equivalent words in the target language, while the meaning of the original version needed to be preserved and retained $(24,27)$. In 
Table 5: Face validity index for the Basic Lifestyle and Demographic Information questionnaire by 30 respondents

\begin{tabular}{lccccc}
\hline \multirow{2}{*}{ Items } & \multicolumn{7}{c}{ Section } \\
\cline { 2 - 6 } & A & B & C & D & Overall \\
\hline S-FVI/Ave & 1.00 & 1.00 & 1.00 & 1.00 & 1.00 \\
S-FVI/UA & 1.00 & 1.00 & 1.00 & 1.00 & 1.00 \\
Proportion Clear/Comprehensible & 1.00 & 1.00 & 1.00 & 1.00 & 1.00 \\
\hline
\end{tabular}

Note: S-FVI (Scale-level face validity index); S-FVI/UA (Universal agreement calculation method); S-FVI/Ave (Averaging calculation method); Average proportion of items judged clear/comprehensible across 30 respondents.

Table 6: Face validity index for the Monthly Health Questionnaire by 30 respondents

\begin{tabular}{|c|c|c|c|c|c|}
\hline \multirow{2}{*}{ Items } & \multicolumn{5}{|c|}{ Section } \\
\hline & A & B & B (I) & B (II) & Overall \\
\hline S-FVI/Ave & 1.00 & 1.00 & 1.00 & 1.00 & 1.00 \\
\hline $\mathrm{S}-\mathrm{FVI} / \mathrm{UA}$ & 1.00 & 1.00 & 1.00 & 1.00 & 1.00 \\
\hline Proportion Clear/Comprehensible & 1.00 & 1.00 & 1.00 & 1.00 & 1.00 \\
\hline
\end{tabular}

Note: S-FVI (Scale-level face validity index); S-FVI/UA (Overall universal agreement calculation method); S-FVI/Ave (Overall averaging calculation method); Overall average proportion of items judged clear/comprehensible across 30 respondents.

the current study, the BLDQ and MHQ were translated from the source language (English) into the target language (Malay). The translation process was performed by local Malay linguistic experts, with cooperation from the investigators, to assure that the Malay translations retained the original meaning of the English version questionnaires. Content validations of the translated version questionnaires in Malay among 10 experts showed good clear understanding of the contents and the languages used. This indicated that the translation process was culturally valid and reliable to be used in the target population. Reliability is referred to the accuracy of a translated version from the angles of semantic equivalence, technical accuracy and textual completeness. It is of utmost importance that the translation is readable, clear and conforms to the grammar and discourse conventions in the translated language. Also, the translated version should be stylistically appropriate, culturally acceptable by the target population and should have the same communicative effect as the source language. A content validity index of 0.8 or more for a new tool is deemed acceptable and applicable (17, 19, 20). In this study, both BLDQ and MHQ showed an overall CVI of over 0.95, indicating the contents were highly relevant to represent the measured outcome and the Malay population. In addition, the original questionnaires (English) and the translated version (Malay) showed a high CVI, indicating similar patterns and good agreement between both English and Malay versions.

The back translated version (from Malay back-translated to English) was subsequently compared with the original source language version (English) by a group comprising of translators and investigators. Upon discussion and reconciliation, only minor amendments were needed, indicating clarity and the absence of discrepancies and/or ambiguities.

Face validity is often employed as an assessing index to demonstrate robustness against scrutiny and to illustrate the level of understanding by the target population towards items in a questionnaire (28). 
An FVI value of 1.0 obtained from the face validity pilot testing involving 30 respondents that represented future potential subjects (parents of pre-school children), indicated the suitability for use among the Malay-speaking population. In addition, the subjects involved in this process were randomly selected, thus minimising selection bias.

\section{CONCLUSIONS}

To our knowledge, this is the first instrument which is developed, translated and validated in Malay, to evaluate the occurrence and prevalence of diarrhoea and respiratory-related illnesses in young children during probiotic administration. This instrument is also simple, easily understood and could be applied to subjects from other age groups with minimal alterations.

\section{CONFLICT OF INTEREST}

The authors declare no conflict of interest. All the authors identified and declared that there is no any personal circumstances or interest that may be perceived as inappropriately influencing the representation or interpretation of reported research results. The founding sponsor had no role in the design of the study; in the collection, analyses, or interpretation of data; in the writing of the manuscript, and in the decision to publish the results.

\section{SUPPORTING INFORMATION}

S1 File: Basic Lifestyle and Demographic Questionnaire (BLDQ) (English version)

S2 File: Monthly Health Questionnaire (MHQ) (English version)

S3 File: Basic Lifestyle and Demographic Questionnaire (BLDQ) (Malay version)
S4 File: Monthly Health Questionnaire (MHQ) (Malay version)

\section{ACKOWLEDGEMENTS}

This work had received financial support from an external grant (304/ PTEKIND/650689) provided by the Morinaga Milk Industry Co., Ltd. and USM Fellowship provided by Universiti Sains Malaysia, Pulau Pinang, Malaysia.

\section{REFERENCES}

1. Xiao JZ, Kondo S, Yanagisawa N, Miyaji K, Enomoto K, Sakoda T, Iwatsuki K, Enomoto T. Clinical efficacy of probiotic Bifidobacterium longum for the treatment of symptoms of Japanese cedar pollen allergy in subjects evaluated in an environmental exposure unit. Allergol Int. 2007;56(1):67-75. https://doi.org/10.2332/ allergolint.O-06-455

2. Xiao JZ, Kondo S, Yanagisawa N, Takahashi $\mathrm{N}$, Odamaki $\mathrm{T}$, Iwabuchi $\mathrm{N}$, Iwatsuki $\mathrm{K}$, Kokubo S, Togashi H, Enomoto K, Enomoto T. Effect of probiotic Bifidobacterium longum BB536 in relieving clinical symptoms and modulating plasma cytokine levels of Japanese cedar pollinosis during the pollen season. A randomized double-blind, placebo-controlled trial. J Investig Allergol Clin Immunol. 2006;16(2):86-93.

3. Hsu VP, Abdul Rahman HB, Wong SL, Ibrahim LH, Yusoff AF, Chan LG, Parashar U, Glass RI, Bresee J. Estimates of the burden of rotavirus disease in Malaysia. J Infect Dis. 2005;192Suppl 1:S80-6. https:// doi.org/10.1086/431494

4. Hung LC, Wong SL, Chan LG, Rosli R, $\mathrm{Ng}$ AN, Bresee JS. Epidemiology and strain characterization of rotavirus diarrhea in Malaysia. Int J Infect. 2006;10(6):470-4. https://doi.org/10.1016/j.ijid.2006.05.008 
5. Cheah WL, Lee PY, Syed Alwi SAR, Kamaruddin K, Albela H, Lau EH, Noraini O, Siti Sanaa WA. Acute gastroenteritis among indigenous paediatric patients - A descriptive study in a rural district hospital, Sarawak. Malaysian Journal of Medicine and Health Sciences. 2011;7:3-7.

6. Hatakka K, Savilahti E, Ponka A, Meurman JH, Poussa T, Nase L, Saxelin M, Korpela R. Effect of long term consumption of probiotic milk on infections in children attending day care centres: double blind, randomised trial. BMJ. 2001;322:1327. https://doi. org/10.1136/bmj.322.7298.1327

7. FAO/WHO. Health and nutritional properties of probiotics in food including powder milk with live lactic acid bacteria. Report of a joint FAO/WHO expert consultation on evaluation of health and nutritional properties of probiotics in food including powder milk with live lactic acid bacteria. [cited 2016 May 15]. Available from: http://www.fao.org/3/a-a0512e.pdf

8. Kondo J, Xiao JZ, Shirahata A, Baba M, Abe A, Ogawa $\mathrm{K}$ and Shimoda T. Modulatory effects of Bifidobacterium longum BB536 on defecation in elderly patients receiving enteral feeding. World J Gastroenterol. 2013;19(14):2162-2170. https://doi. org/10.3748/wjg.v19.i14.2162

9. Guandalini S. Probiotics for prevention and treatment of diarrhea. J Clin Gastroenterol. 2011;45:S149-53. https://doi.org/10.1097/ MCG.0b013e3182257e98

10. Lehtoranta LM, Pitkäranta A, Korpela R. Probiotic Lactobacillus Rhamnosus GG and respiratory illness in children. Dietary Fibres \& Pre/Probiotics. 2012; 23(6):4-7.

11. Pan Y. de la Puente M. Census Bureau Guideline for the translation of data collection instruments and supporting materials: documentation on how the guideline was developed. Washington DC: Statistical Research Division, U.S. Bureau of the Census; 2005.
12. Cook DA. Beckman TJ. Current concepts in validity and reliability for psychometric instruments: theory and application. Am J Med. 2006;119(2):166.e7-16. https://doi. org/10.1016/j.amjmed.2005.10.036

13. Streiner DL, Norman GR. Health measurement scales: a practical guide to their development and use. New York: Oxford University Press Inc.; 2008. https://doi.org/10.1093/ acprof:oso/9780199231881.001.0001

14. Dyrbye LN, Szydlo DW, Downing SM, Sloan JA, Shanafelt TD. Development and preliminary psychometric properties of a well-being index for medical students. BMC Medical Education. 2010;10:8. https://doi. org/10.1186/1472-6920-10-8

15. Thompson M, Vodicka TA, Blair PS, Buckley DI, Heneghan C, Hay AD. Duration of symptoms of respiratory tract infections in children: systemic review. BMJ. 2013;347 (f7027):1-9. https://doi.org/10.1136/bmj. f7027

16. WGO World Gastroenterology Organisation practice guideline: Acute diarrhea. [cited 2016 April 17). Available from: http://doctorru.org/main/1100/1101.pdf

17. Polit DF, Beck CT. The content validity index: are you sure you know what's being reported? Critique and recommendations. Research in Nursing \& Health. 2006;29(5):489-97. https://doi.org/10.1002/ nur.20147

18. International Consultation on Incontinence Modular Questionnaire (ICIQ). Validation protocol. (2014; cited 2016 March 15). Available from: http://www.iciq.net/ validationprotocol.htm

19. Polit DF, Beck CT, Owen SV. Is the CVI an acceptable indicator of content validity? Appraisal and recommendations. Research in Nursing \& Health. 2007;30(4):459-67. https://doi.org/10.1002/nur.20199 
20. Davis LL. Instrument review: Getting the most from a panel of experts. Applied Nursing Research. 1992;5:194-7. https://doi. org/10.1016/S0897-1897(05)80008-4

21. Harkness JA. Schoua-Glusberg A. Questionnaires in translation. ZUMANachrichten Spezial. 1998:40.

22. Sperber AD, Develis RF, Boehlecke B. Cross-cultural translation: methodology and validation. Journal of Cross-cultural Psychology. 1994;25(4):501-24. https://doi. org/10.1177/0022022194254006

23. Sousa VD, Rojjanasrirat W. Translation, adaptation and validation of instruments or scales for use in cross-cultural health care research: a clear and user-friendly guideline. Journal of Evaluation in Clinical Practice. 2011;17:268-74. https://doi.org/10.1111/ j.1365-2753.2010.01434.x

24. Su CT, Parham LD. Generating a valid questionnaire translation for cross-cultural use. The American Journal of Occupational Therapy. 2002;56:581-5. https://doi.org/ 10.5014/ajot.56.5.581
25. Laerd Dissaertation. Face validity. Available from: http://dissertation.laerd.com/facevalidity.php (2012; cited 2016 March 27).

26. Banville D, Desrosiers P, Genet-Volet Y. Translating questionnaires and inventories using a cross-cultural translation technique. Journal of Teaching in Physical Education. 2000;19:374-87. https://oi.org/10.1123/ jtpe.19.3.374

27. Lee YY, Waid A, Tan HJ, Chua SBA, Whitehead WE. Validity and reliability of the Malay-language translation of the Rome III diagnostic questionnaire for irritable bowel syndrome. Journal of Gastroenterology and Hepatology. 2012;27:746-50. https://doi. org/10.1111/j.1440-1746.2011.06943.x

28. Shuttleworth M. Face validity. (2008-2017; cited 2016 March 25). Available from: https://explorable.com/face-validity 